\title{
A Consideration of Learning Cellular Automata with The Global Self-Improving Behavior
}

\author{
Fei Qian† and Hironori Hiratał \\ † Department of Computer Science, Hiroshima-Denki Institute of Technology \\ 6-20-1 Nakano Aki-ku, Hiroshima, Japan, 739-03 \\ Emailfei@mercury.c.hiroshima-dit.ac.jp \\ $\ddagger$ Department of Electrical and Electronics Engineering, Chiba University \\ 1-33 Yayoi-cho Inage-ku Chiba, Japan, 263 \\ Email hiro@cute.te.chiba-u.ac.jp
}

\begin{abstract}
We present a learning cellular automaton model (LCA) that is an extended generation of the traditional cellular automata, with some self-improving functions. The selfimproving learning cellular automaton consists of two parts: the main body and the universal constructor, i.e. a learning cellular automaton can constructs itself through the use of a constructing arm, and it also makes any configurations whose description can be stored on its input tape.

We aim at using this model to solve some combinatorial optimization problems in some environments with non-complete prior information.
\end{abstract}

\section{Introduction}

In complex adaptive systems interesting global behaviors emerge from many local interactions. When the emergent behavior is a computation, we refer to it as an emergent computation. The premise of the emergent computation is that interesting and useful computational systems can be constructed by exploiting interactions among primitive components. The research on self-improving or self-reproducing behavior may play an important role in the emergent computation,

In the self-reproducing cellular automata of von Neumann ${ }^{[l]}$, the copy is created by interpreting the coded description on the tape as a sequence of the instructions which cause the construction, cell by cell, of new machine in an unoccupied part of cellular space. The copying actually occurs at the moment when the state of some previously quiescent cells immediately adjacent to the tip of the constructing arm is changed to the desired new state. To judge the copy, the free energy must be supplied to the system to enable it to change states. The force that causes the change of state corresponds to the free energy of the system.

In particular, von Neumann demonstrated the possibility of the self-replicability of a computational structure by actually designing a self-reproducing automaton consisting of two-dimensional cellular arrangement with a large number of 29-state automata cells. The next state of 29-state automaton is a function of its own current state and the state of its four neighbors in the two-dimensional cellular space. Within this framework, von Neumann was able to conceive a self-reproducing automaton endowed with the properties of both computational and constructional universality. Unfortunately, the automaton is so complex that notwithstanding further simplifications, even today's state-of-the-art computers lack the power to simulate it in entirety.

In here, we present a learning cellular automaton model (LCA) that is an extended generation of the traditional cellular automata, with some self-improving functions, called as SILCA. We aim at using this model to solve some combinatorial optimization problems in some environments with non-complete prior information (e.g. a TSP with no size information, etc.).

\section{The structure of the self-improving learning cellular automata}

The self-improving learning cellular automaton consists of two parts: the main body and the universal constructor, i.e. learning cellular automaton capable of constructing, through the use of a constructing arm, any con- 
figurations whose description can be stored on its input tape. Using the constructing arm, automaton is able to construct a copy (an offspring) of itself. From the micro view point, this consideration is the same as von Neumann's self-reproducing automaton, but from the global view point the structure and the function are just selfimproving.

The self-improving learning cellular automaton is defined as a stochastic cellular automaton $A$ containing a universal constructor $W$, with its own random environment $E$. Here,

$$
\begin{aligned}
A & =\{U, X, Y, Q, \xi, F, O, T, W\} \\
E & =\{Y, C, r\}
\end{aligned}
$$

where,

$$
\begin{aligned}
& U: \text { The cellular space. } U=\left\{u_{j}, j=1,2, \ldots N\right\} \\
& X \quad: \text { The set of inputs. } X=\left\{x_{j}, 0 \leq j<\infty\right\} \\
& Y: \text { The set of outputs. } Y=\left\{y_{j}, 0 \leq j<\infty\right\} \\
& Q \quad: \text { The set of internal states. } Q=\left\{q_{j}, 0 \leq j<\infty\right\} \\
& \xi \quad: \text { The neighborhood state configuration function. } \xi: U \rightarrow \Omega, \Omega \subseteq U . \\
& F \quad: \text { The stochastic state transition function. } F: Q \times X \times r \rightarrow Q . \\
& O \quad: \text { The stochastic output function. } O: Q \rightarrow Y . \\
& T: \text { The reinforcement scheme. } Q(t+1)=T(Q(t)) . \\
& W \quad: \text { The constructor. } W=\left\{W_{c}, W_{f}\right\} . \\
& C \quad: \text { The penalty probability distribution. } C=\left\{c_{j}, 0 \leq j<\infty\right\} \\
& r \quad: \text { The reinforcement signal. } r=\left\{r_{j}, 0 \leq j<\infty\right\}
\end{aligned}
$$

The random environment $E$ is assumed to be stationary and is characterized by the set of penalty probability distribution $C$. For all $c^{j} \in C$,

$$
c_{k}^{j}=\operatorname{prob}\left(r=-11 y^{j}=y_{k}^{j}, x^{j}\right)
$$

Here, we will confine our attention to S-model, i.e. for the case $r=\left\{r_{j}, 0 \leq j<\infty\right\}$. Where the $r_{j}$ represents the strength of the penalty responses.

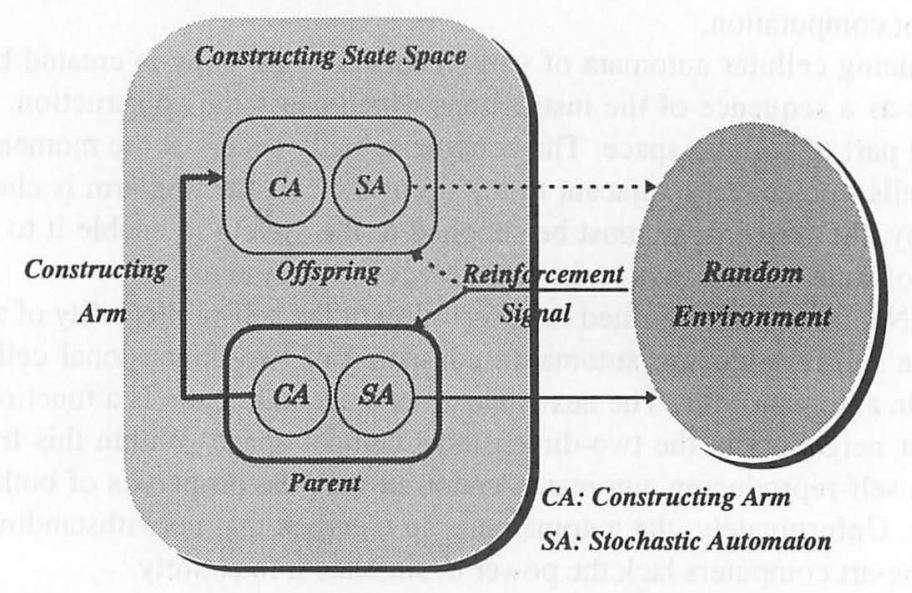

\section{Fig.1. The learning cellular automaton with self-Improving}

For constructor $W$, where, $W_{c}$ is the constructing state space, $W_{c}=\left\{s_{i j}, i, j=0,1, \ldots\right\}$, in here, the state $s_{i j}$ is respectively defined as 0 for quiescent state; 1 , sheath state; 2 , activation state; 3 , construction state; 4 , destruction; $d$, date transmission state. $W_{f}$ is the function control unit. 


\section{The constructing state space and the universal constructor}

To make an account of the constructing state space and the universal constructor, there are some representations provided by von Neumann, C.G. Langton, E.F. Codd and other researchers.

Langton's "periodic emitter" ${ }^{[2]}$ is based on Codd's universal constructors ${ }^{[3]}$. The constructor (automaton) (Fig. 2) is essentially a duplicated square loop called as internal and external sheath. The state necessary for the construction of duplicate loop circulate counterclockwise. A reproduction is achieved by extending a constructing arm to the left at regular interval corresponding to the size of one side of the loop. After such runs the constructing arm will have folded upon itself. When the new constructing loop is closed, the constructing arm will be cut down and the new offspring with new constructing loop will be obtain. Finally, the parent loop will die when all offspring of itself in another direction has reproduced. The constructor will replicate itself to fill the available constructing state space, when one gives it sufficient time.

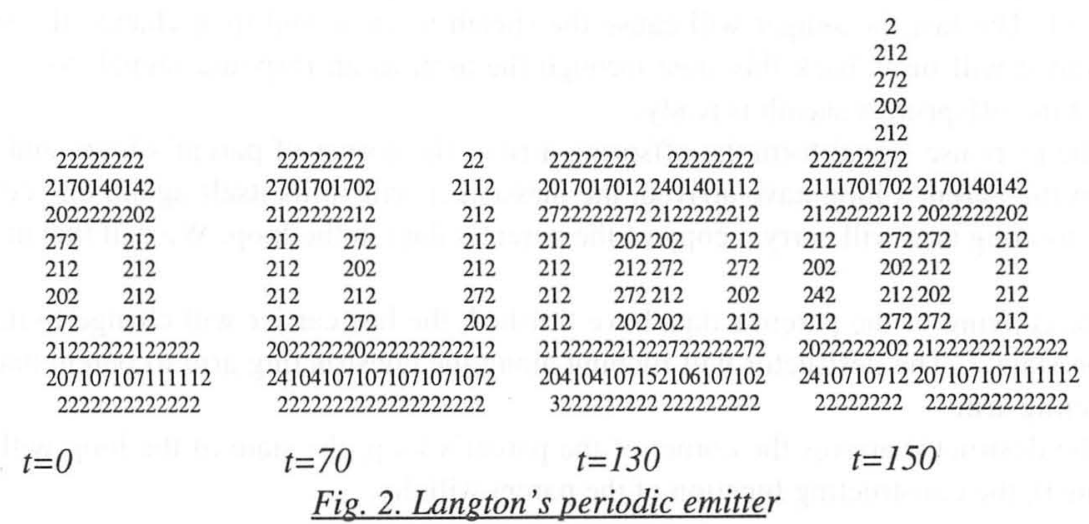

In here, our consideration is similar to Langton's loop. For each cell of constructor we use a 9-neighborhood (contain itself also) and use the internal sheath only. In our internal sheath, we have two (Fig.3.) or four (Fig.4.) out-going cells at the two (left-top and right bottom) or four corners of constructor. Initially, these two or four cells are in the open state, and will change to the closed state once the offspring is accomplished.

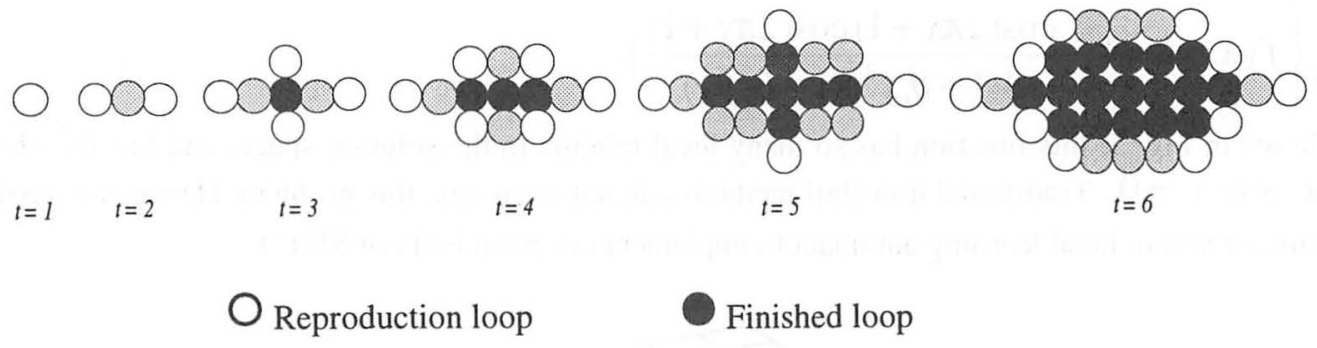

Fig. 3 The two out-going cells loop and its generations

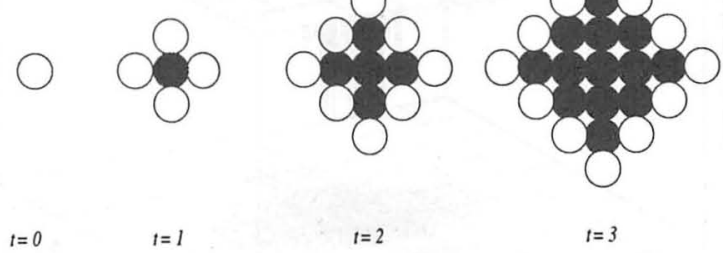

Fig.4 The four out-going cells loop and its generations

The initial configuration is in the form of a square loop wrapped around a sheath. The size of the loop is variable. We can use any size of the loop, for an example $8 \times 8$. Near the out-going cells of the loop, the initial 
state is in the sheath state 1 . The automaton should attempt to duplicate itself in all out-going directions with following rule.

\section{Reproducing rule $R_{5,4}{ }^{1)}$ :}

(1) Once the constructor starts, operating the constructing date starts running around the loop.

(2) When the sheath state 2 which we call as messenger reaches a out-going cell and it finds the cell open, the messenger splits into two same cells. One cell whose left cell is in destruction state continues turning around the loop, and the other one starts extending the constructing arm.

(3) Once the constructing arm has start extending, each messenger that arrives to out-going cells will again split and one of the parent's copies will send though the arm.

(4) Once the first messenger reaches the tip of the constructing arm, the state of the tip changes to activation state 3 . The constructing arm will start the offspring's sheath constructing.

(5) The next messengers will turn the tip of the constructing arm to the left, until the four corners have completed. The last messenger will cause the sheath to close and then change the state to activation state. And it will turns back this state through the arm, as an response signal, to response the parent loop that the offspring's sheath is ready.

(6) When the response signal form the offspring arrives the corner of parent's loop, and the next messenger from the parent's loop have arrived, the messenger will splits itself again, one copy running along the constructing arm will carry a copy of the parent's data in the loop. We call this messenger as a carrier.

(7) Once the copying of the parent's data have finished, the last carrier will change to destructor with destruction state 4 . The destructor will running along the constructing arm to parent and break down the constructing arm.

(8) When the destructor arrives the corner of the parent's loop, the state of the loop will change to quiescent state 0 , the constructing function of the parent will die.

\section{Design the main body to solve some functional optimization problems} problem.

To show how the self-reproducing learning cellular automata works, we consider the following optimization

$$
\min _{x, y \in[-20,20]}\left\{f(x, y)=1-\frac{\cos (2 \pi x+1) \cos (2 \pi y+1)}{4\left(1+\theta_{1} x^{2}\right)\left(1+\theta_{2} y^{2}\right)}\right\}
$$

As shown in Fig. 5, this function has so many local minima in the solution space, and has the global minimum 0 at $x=y=0$. Traditional downhill methods can not overcome this problem. Hence, we design a variable structure of hierarchical learning automata to implement our main body of SILCA.

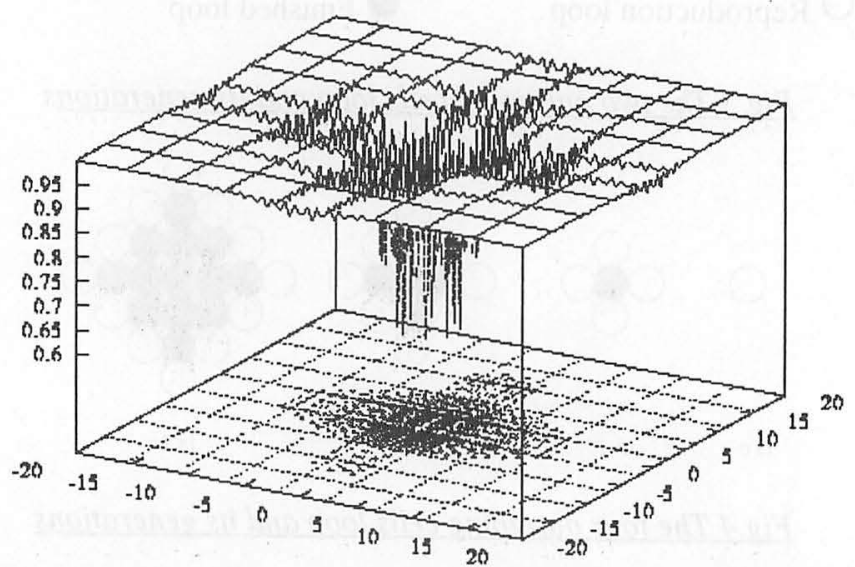

Fig. 5 The object function and its distribution of the local minima.

\footnotetext{
1) The rule for 5-states and 4-out-going cells.
} 
To solve this problem we use the reinforced random search method described as follows.

The value areas of $x$ and $y$ are represented by

$$
L=\{\underbrace{l_{0}^{x}, l_{1}^{x}, \ldots, l_{n x}^{x}}_{\text {for } x} \underbrace{l_{0}^{y}, l_{1}^{y}, \ldots, l_{n y}^{y}}_{\text {for } y}\}, \quad l_{i}^{j} \in\{0,1\}, i=0,1, \ldots, n j ; j=x, y
$$

Where, the $l_{i}^{j}$ is depend on the encoding method, we use the gray code encoding method to encode the $x$ and $y$ to the vector $L$. Therefore, $G R A Y^{-1}\left(l_{0}^{x}, l_{1}^{x}, \ldots, l_{n x}^{x}\right), G R A Y^{-1}\left(l_{0}^{y}, l_{1}^{y}, \ldots, l_{n y}^{y}\right) \in[-20,20], G R A Y^{-1}(l)$ is an gray decoding function that decodes the vector 1 to a scalar value. levels.

The variable structure of hierarchical learning automata (VSHLA) is shown as Fig. 6, constructed as three

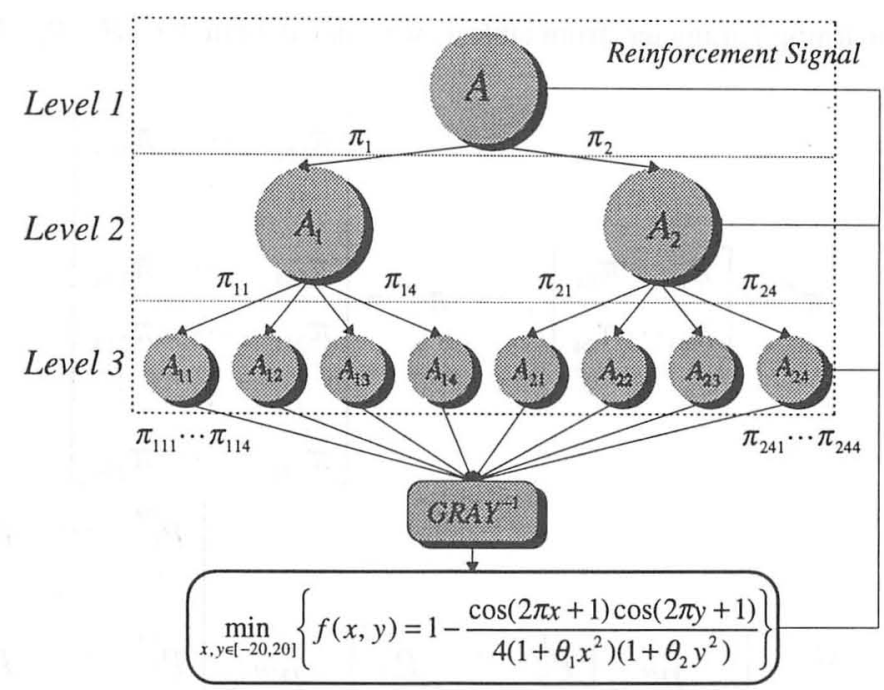

\section{Fig.6. The variable structure of hierarchical learning automata for functional optimization problems}

On the first level there is one stochastic automaton with two actions $y \in\left\{y^{1}, y^{2}\right\}$. When the automaton on the first level selects the action $y^{i}(i=1,2)$ at the time $n$, i.e. $y(n)=y^{i}$, the $i t h$ automaton on the second level will be fired. The automata on the second level has four actions, which fire the automata on the third level. For the automata on the third level, the action are corresponding to the subset of a gray encoded vectors of $x$ and $y$.

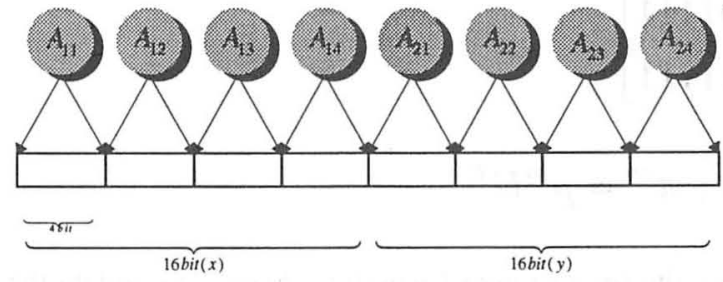

\section{Fig. 7. Reinforced random search area for each automaton.}

\section{Definition:}

First level: $A=\{y, r, \phi, p, o, T\}$, where, $y \in\left\{y_{1}, \cdots, y_{4}\right\}$ is the action (output), $r$ is the input, $\phi$ is the state set, $p=\left\{p_{1}, \cdots, p_{4}\right\}$ is the probability distribution on the action set.

Second level: $A_{i}=\left\{y^{i}, r, \phi^{i}, p^{i}, o^{i}, T^{i}\right\}, i=1,2$, where $y^{i} \in\left\{y_{1}^{i}, \cdots, y_{16}^{i}\right\}, p^{i}=\left\{p_{1}^{i}, \cdots, p_{16}^{i}\right\}$, 
Third level: $A_{i j}=\left\{y^{i j}, r, \phi^{i j}, p^{i j}, o^{i j}, T^{i j}\right\}, i=1,2 ; j=0, \cdots, 3$, where, $y^{i j} \in\left\{y_{1}^{i j}, \cdots, y_{16}^{i j}\right\}$,

$$
p^{i j}=\left\{p_{1}^{i j}, \cdots, p_{16}^{i j}\right\} \text {. }
$$

On our simulations we encode the $x$ and $y$ as 16-bit 0-1 vectors, i.e. in the eq. (5) $n x=15 n y=15$, and split each vector into four pieces (Fig.7). Therefore, the random search is implemented as the table 1.

Table 1. The meaning of each action

\begin{tabular}{|c|c|c|c|c|c|c|c|c|c|c|c|c|}
\hline \hline level & \multicolumn{9}{|c|}{1} & \multicolumn{9}{|c|}{2} & \multicolumn{4}{|c|}{3} \\
\hline \hline$y$ & $y_{1}$ & $y_{2}$ & $y_{3}$ & $y_{4}$ & $y_{1}^{i}$ & $y_{2}^{i}$ & $\cdots$ & $y_{16}^{i}$ & $y_{1}^{i j}$ & $y_{1}^{i j}$ & $\cdots$ & $y_{1}^{i j}$ \\
\hline update & - & $A_{1}$ & $A_{2}$ & $A_{1}, A_{2}$ & - & $A_{1}^{i}$ & $\cdots$ & $A_{1}^{i}, \cdots A_{4}^{i}$ & bit 0 & bit 1 & $\cdots$ & bit $0 \ldots$ bit4 \\
\hline \hline
\end{tabular}

Each bit on the $L$ is turns around 0 and $l$ with the probability

$$
m_{i j k}=\lambda_{1} \pi_{i} \cdot \lambda_{2} \pi_{i j} \cdot \lambda_{3} \pi_{i j k}, i=1,2, j=1,2,3,4, k=1,2,3,4 \text {. }
$$

where, $0<\lambda<1$ is a learning parameter, from table 1 , we can calculate the $\pi_{i}, \pi_{i j}, \pi_{i j k}$ as follows. Let

$$
\begin{aligned}
\pi^{\prime} & =\left[\begin{array}{ll}
\pi_{1} & \pi_{2}
\end{array}\right]^{T}, \quad \pi^{\prime \prime}=\left[\begin{array}{l}
\pi_{11} \cdots \pi_{14} \\
\pi_{21} \cdots \pi_{24}
\end{array}\right], \quad \pi^{\prime \prime \prime}=\left[\begin{array}{ccc}
\pi_{111} & \cdots & \pi_{114} \\
\vdots & \vdots & \vdots \\
\pi_{141} & \cdots & \pi_{144} \\
\pi_{211} & \cdots & \pi_{214} \\
\vdots & \vdots & \vdots \\
\pi_{241} & \cdots & \pi_{244}
\end{array}\right] \\
P^{\prime} & =\left[\begin{array}{cc}
p_{2}+p_{4} & 0 \\
0 & p_{3}+p_{4}
\end{array}\right],, P^{\prime \prime}=\left[\begin{array}{lll}
p_{1}^{1} & \cdots & p_{16}^{1} \\
p_{1}^{2} & \cdots & p_{16}^{2}
\end{array}\right], P^{\prime \prime \prime}=\left[\begin{array}{ccc}
p_{1}^{10} & \cdots & p_{16}^{10} \\
\vdots & \cdots & \vdots \\
p_{1}^{13} & \cdots & p_{16}^{13} \\
p_{1}^{20} & \cdots & p_{16}^{20} \\
\vdots & \cdots & \vdots \\
p_{1}^{23} & \cdots & p_{16}^{23}
\end{array}\right] \\
U & =\left[\begin{array}{l}
0101010101010101 \\
0011001100110011 \\
0000111100001111 \\
0000000011111111
\end{array}\right]
\end{aligned}
$$

then,

$$
\pi^{\prime}=p^{\prime}, \pi^{\prime \prime}=p^{\prime \prime} U^{T}, \pi^{\prime \prime \prime}=p^{\prime \prime \prime} U^{T}
$$

For each automaton we use the linear reward-penalty scheme ${ }^{[4]}$ to update the action probabilities.

Our simulations are start with one automaton that includes a universal constructor with two out-going cells and a VSHLA. We set the searching area $[a, b] \in[-20,20]$ randomly. Once the state transition on the constructing loop has visited an out-going cell, we can get the following two processes.

- Sequential process: the local searching on the parent is finished and a local minimum is found. Then the parent automaton begin to construct two offspring on the other areas and play the same things on the offspring;

- Partially parallel process: the parent constructs an offspring and carry out the local searching independently. 
Here, the simulation results for partially parallel process method are shown in Fig. 8 and Fig. 9. The simulation is start on the area $[-7,-5]$ with one SILCA. The size of the inner sheath loop is set to $1 / 5$ simulation times for one offspring. After 10 generation, the whole local minimums have found. The Fig. 8 shows the evolution of probabilities of the offspring that near the global minimum area. From Fig. 8 we can see, when the random searching reaches to the global minimum area, the convergence probability for the non-action output becomes higher than others, therefore, the searching process will be finished. Because the probabilities of the another actions are not equal to zero, the scheme has some oscillatory properties. The Fig.9(a) shows the convergence corresponding to various learning parameters(the $\theta$ is reward parameter of the linear reward-inaction scheme), Fig.9(b) shows the local minimums found by each offspring. The results show that using the SILCA we can search the full solution space to find the optimum.

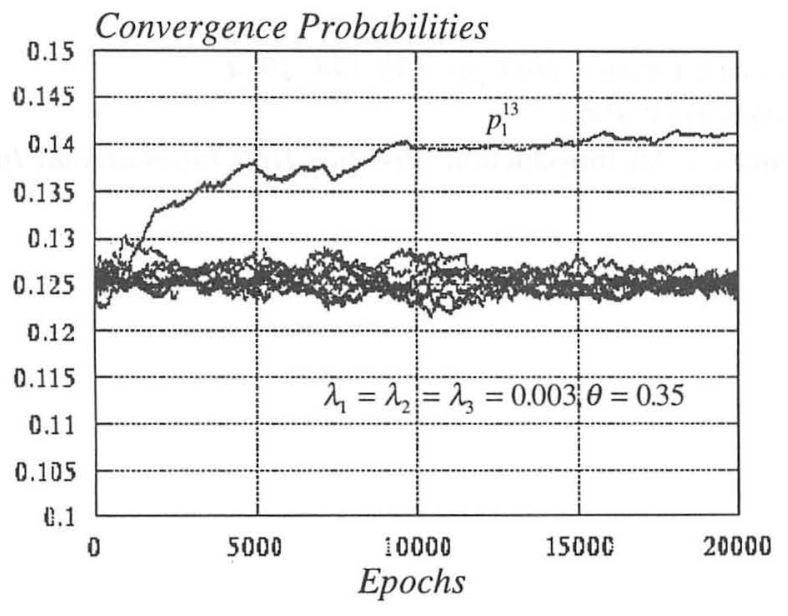

(a)

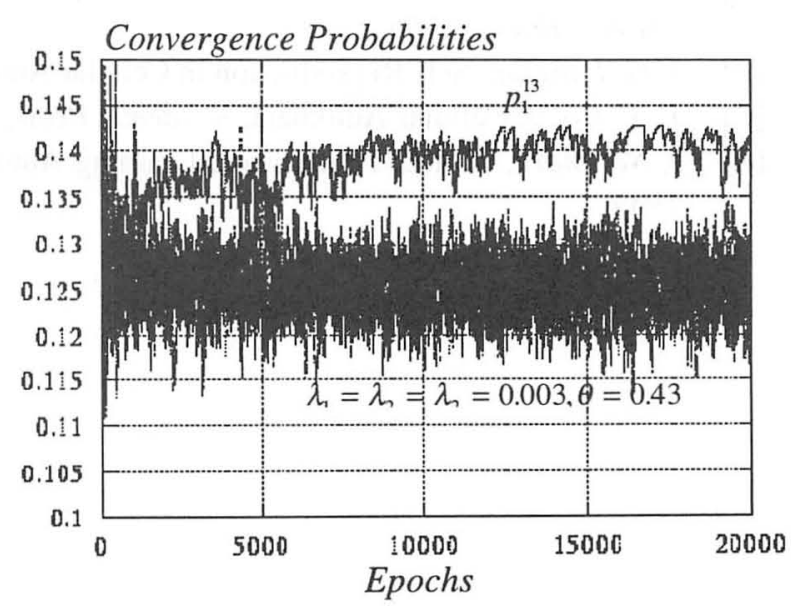

(b)

Fig. 8 Evolution of the stochastic automaton's probabilities on the third level

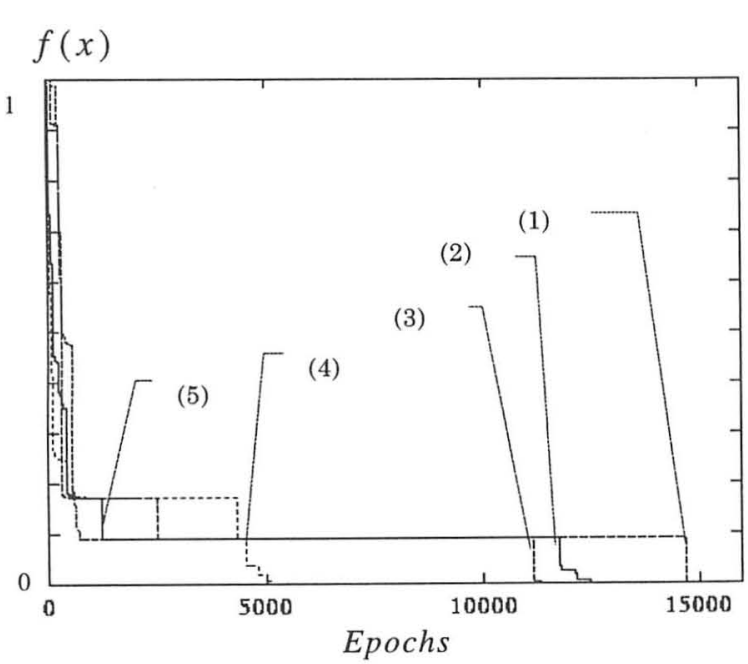

(a)

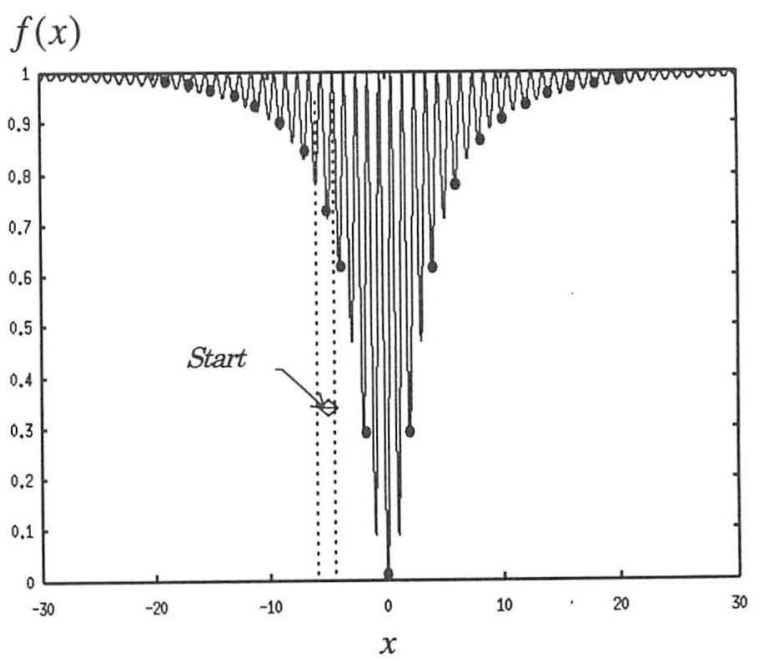

(b)

Fig. 9 (a)Convergence corresponding to various learning parameter

(1) $\lambda_{1}=\lambda_{2}=\lambda_{3}=0.001, \theta=1$, (2) $\lambda_{1}=\lambda_{2}=\lambda_{3}=0.003, \theta=1$, (3) $\lambda_{1}=\lambda_{2}=\lambda_{3}=0.003, \theta=0.6$

(4) $\lambda_{1}=0.001, \lambda_{2}=0.002, \lambda_{3}=0.001, \theta=0.45$, (5) $\lambda_{1}=0.001, \lambda_{2}=0.015, \lambda_{3}=0.002, \theta=53$

(b) The local minima found by each offspring

\section{Conclusions}


We have presented the self-improving learning cellular automaton (SILCA). The SILCA consists of two parts: the main body that are implemented by the variable structure of hierarchical learning automata using the reinforced random search method, and the universal constructor implemented by the simplified Langton's peri-' odic emitter.

Because using the SILCA we can search the solution space carefully and each offspring can carry out the local searching parallel, we can use the parallel computer to implement the SILCA and solve the practical functional optimization problems quickly.

\section{Reference}

[1] J. von Neumann, The Theory of Self-Reproducing Automata, A. W. Burks, ed,. University of Illinois Press, Urbana, 1966.

[2] C.G. Langton, Self-Reproduction in Cellular Automata, Physics, 10D, pp. 135-144, 1984

[3] E. F. Codd, Cellular Automata, Academic Press, New York, 1968.

[4] K.Narendra, M.A.L. Thathachar, Learning Automata - An Introduction, Prentice-Hall International Inc. 1989. 